\title{
Simulation and measurement of transcranial near infrared light penetration
}

\author{
Lan Yue ${ }^{\mathrm{a}}$, Manuel Monge ${ }^{\mathrm{b}}$, Mehmet H. Ozgur ${ }^{\mathrm{c}}$, Kevin Murphy ${ }^{\mathrm{d}}$, Stan Louie ${ }^{\mathrm{e}}$, Carol A. Miller ${ }^{\mathrm{f}}$, Azita \\ Emami $^{\mathrm{b}}$, Mark S. Humayun ${ }^{\mathrm{a}}$ \\ ${ }^{a}$ Dept. of Ophthalmology, USC Eye Institute, University of Southern California, Los Angeles, CA, \\ USA 90033; ${ }^{b}$ Dept. of Electrical Engineering, California Institute of Technology, Pasadena, CA, \\ USA 91125; ${ }^{\circ}$ Providence Holy Cross Medical Center, Mission Hills, CA; ${ }^{\mathrm{d}} \mathrm{Sch}$ ool of Medicine; \\ ${ }^{\mathrm{e}}$ School of Pharmacy; ${ }^{\mathrm{f}}$ Dept. of Pathology, University of Southern California, Los Angeles, CA, \\ USA 90033
}

\begin{abstract}
We are studying the transmission of LED array-emitted near-infrared (NIR) light through human tissues. Herein, we simulated and measured transcranial NIR penetration in highly scattering human head tissues. Using finite element analysis, we simulated photon diffusion in a multilayered 3D human head model that consists of scalp, skull, cerebral spinal fluid, gray matter and white matter. The optical properties of each layer, namely scattering and absorption coefficient, correspond to the $850 \mathrm{~nm}$ NIR light. The geometry of the model is minimally modified from the IEEE standard and the multiple LED emitters in an array were evenly distributed on the scalp. Our results show that photon distribution produced by the array exhibits little variation at similar brain depth, suggesting that due to strong scattering effects of the tissues, discrete spatial arrangements of LED emitters in an array has the potential to create a quasi-radially symmetrical illumination field. Measurements on cadaveric human head tissues excised from occipital, parietal, frontal and temporal regions show that illumination with an $850 \mathrm{~nm}$ LED emitter rendered a photon flux that closely follows simulation results. In addition, prolonged illumination of LED emitted NIR showed minimal thermal effects on the brain.
\end{abstract}

Keywords: near-infrared light, transcranial penetration, LED array, finite element analysis

\section{INTRODUCTION}

Optical approaches in laboratory and clinical studies of neurological activities have received increasing attention in recent years. As a result, a variety of optical brain imaging modalities that reflect, for instance, oxygenation and hemodynamics of the brain have been developed for the visualization of the microscopic structure and cortex activation. However, due to the strong scattering of light by biological tissues, optical imaging typically suffers low resolution and short light penetration depth, limiting its use in adults in clinical settings. Therefore near-infrared (NIR) light is usually employed for improved penetration into the brain. In addition to finding application in optical imaging, NIR light also exhibits therapeutic potentials for neurological disorders. Transcranial irradiation of NIR light is shown to produce beneficial outcomes for ischemic stroke patients[1] and it was reported to improve cognitive function, sleep and posttraumatic stress disorder symptoms of the traumatic brain injury patients, likely by enhancing ATP metabolism in compromised cells[2]. Despite significant improvements from visible light, transcranially applied NIR light still undergoes significant attenuation as it passes through the multiple overlying tissues of an adult human head such as scalp and skull, with only a very small fraction of light reaching the brain. To obtain a better view of transcranial NIR light penetration, we hereby measured light passage through fresh cadaveric human head tissues and investigated the possibility of using NIR LED array that contains multiple emitters to achieve enhanced photon flux in the brain.

Optical Interactions with Tissue and Cells XXVI, edited by E. Duco Jansen, Proc. of SPIE Vol. 9321, 93210S @ 2015 SPIE · CCC code: 1605-7422/15/\$18 - doi: 10.1117/12.2077019 


\section{MEASUREMENT OF TRANSCRANIAL NIR LIGHT PENETRATION}

\subsection{Experimental methods}

Four cadaver heads, 1-3 days postmortem, were tested. Subjects ranged in ages 52 to 67 years who had no history of CNS disorders as verified by gross and microscopic neuropathological examination of cerebral sections. The microscopic evaluation showed no evidence of inflammatory infiltrates, acute or chronic neurodegeneration, edema, or vasculopathies. Tissue samples from four regions: occipital, parietal, temporal and frontal lobe, were obtained using external landmarks for correct localization in the brain. A rotary bone cutter fully penetrates and isolates a $3.8 \mathrm{~cm}$ circular segment of cranial bone. Overlying scalp and underlying dura were cut and an underlying gray and white matter, were removed by a scalpel. Excised tissue layers were assembled in order (external to internal) and placed in a beaker $(100 \mathrm{~mL})$. Thickness of each tissue layer at all four cortical lobar regions was measured.

An $850 \mathrm{~nm}$ LED driven by our custom-made circuit (output power set at $495 \mathrm{~mW}$ ) was positioned $7.5 \mathrm{~cm}$ away from the photodetector, and the tested sample oriented in the light pathway with the scalp layer facing the LED and the brain facing the photodetector, mimicking the actual light penetration from outside to the inside of a skull.

\subsection{Experimental results}

Measurement of the samples excised from the four cranial regions demonstrate an overall slightly greater scalp and skull thickness in the occipital area, though the difference is not significant (Figure 1, Left Panel). The dural layer ranged between 1-2 $\mathrm{mm}$ in all samples. Due to the difficulty of maintaining consistent shape/thickness of the fresh cadaveric brain, non-trivial inter-sample variations in the brain thickness $(1-4 \mathrm{~cm})$ existed. Despite such variations, NIR light that penetrated through samples from various cranial regions underwent somewhat similar levels of attenuation. As shown in Figure 1, right panel, after passing through the scalp, skull, dura, and penetrating 1-4 $\mathrm{cm}$ into the brain $(3-6 \mathrm{~cm}$ overall tissue thickness), light exited from all samples underwent a $\sim 3-4$ orders of magnitude stronger attenuation in comparison with passing through the air alone. The temporal area shows only a slightly and non-significantly weaker attenuation ( $p$ $=0.4$ between temporal and occipital data). These results thus indicate that changes in the placement of an LED emitter from one cranial region to another will be unlikely to result in a large fluctuation in photon penetration, suggesting the possibility of using similarly powered multiple LED emitters placed on various cranial locations to produce a qausisymmetrical light field in the brain.
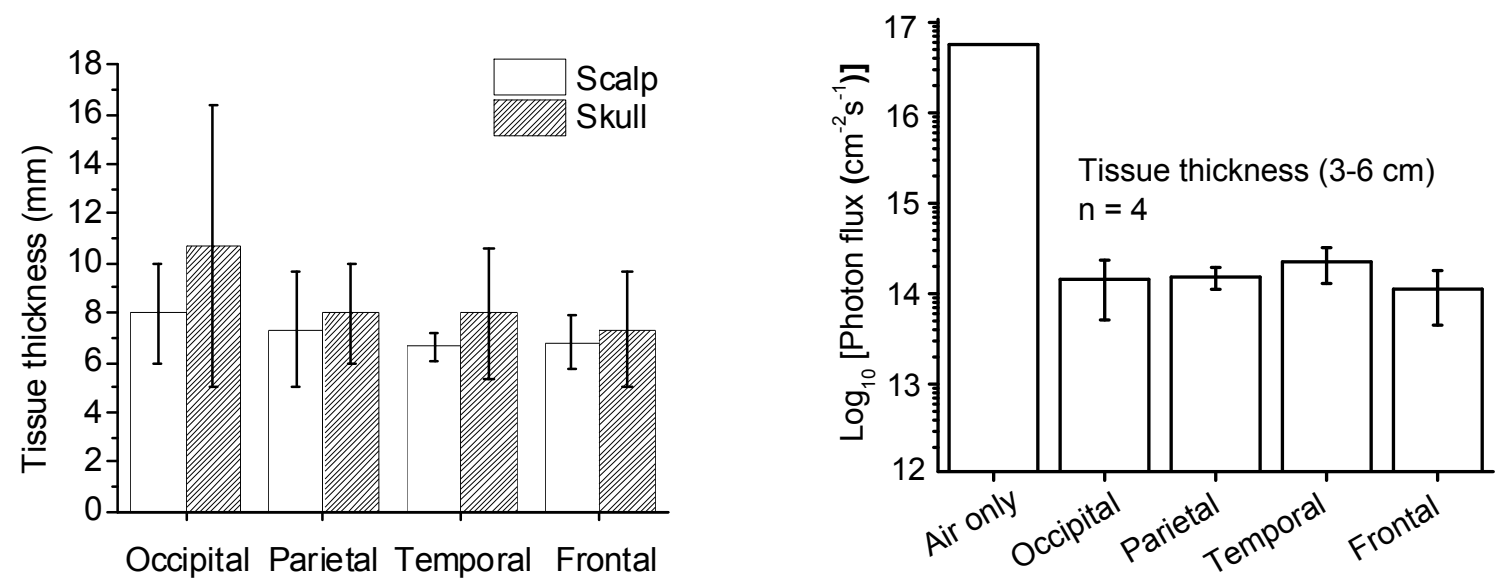

Figure 1. Measurement of $850 \mathrm{~nm}$ NIR light transcranial penetration in the occipital, parietal, temporal and frontal regions of four cadaveric human heads. Left: Measured tissue thickness of the excised scalp and skull; Right: photon flux of the NIR light penetrated through reconstructed tissue blocks. 


\section{SIMULATION OF THE TRANSCRANIAL NIR LIGHT PENETRATION}

\subsection{Background and methods}

To further investigate the feasibility of using multiple LED emitters for improved transcranial penetration, we implemented FEM (finite element method) to simulate the light propagation from a scalp applied LED array. Light attenuation in biological tissues is largely contributed by two mechanisms: absorption and scattering. The diffusion approximation of the radio transfer equation[3] has been widely used to describe the light propagation in highly scattering biological tissues, under the assumption that the absorption coefficient is considerably smaller than the scattering coefficient and that the light propagation is isotropic. The former assumption is generally true with human head tissues and to make the latter valid, we took anisotropic scattering into account of the isotropic diffusion model by modifying scattering coefficient $\mu_{\mathrm{s}}$ to the transport scattering coefficient $\mu_{\mathrm{s}}{ }^{\prime}=(1-\mathrm{g}) \mu_{\mathrm{s}}$, where $\mathrm{g}$ is the anisotropic factor. The time-independent diffusion equation is thus given by:

$$
-\nabla \cdot \kappa(\hat{\mathrm{r}}) \nabla \Phi(\hat{\mathrm{r}})+\mu_{\mathrm{a}}(\hat{\mathrm{r}}) \Phi(\hat{\mathrm{r}})=\mathrm{q}_{0}(\hat{\mathrm{r}})
$$

where at $\hat{r}, \Phi$ is the photon fluence rate, $\kappa$ is the diffusion coefficient $\left[3\left(\mu_{\mathrm{a}}+\mu_{\mathrm{s}}{ }^{\prime}\right)\right]^{-1}, \mu_{\mathrm{a}}$ is the absorption coefficient and $\mathrm{q}_{0}$ is the isotropic source distribution. To solve this diffusion equation, the boundary conditions were defined. The photon diffusion at the head-source interfaces were constrained by the surface Robin boundary condition:

$$
\Phi(\hat{\mathrm{r}})+2 \mathrm{~A} \kappa(\hat{\mathrm{r}}) \hat{\mathrm{n}} \cdot \nabla \Phi(\hat{\mathrm{r}})=-4 \Gamma_{\mathrm{s}}
$$

where $\Gamma_{\mathrm{s}}$ is the strength of the source current and $\mathrm{A}=2.94$ for air-tissue boundary, due to mismatched refractive indices[3]. At the tissue-tissue interfaces, fluence continuity in the normal direction was assumed.

A finite element model was then constructed using COMSOL Multiphysics 4.2 to estimate the photon distribution in a multi-layered 3-D domain representing human head. The geometry of the human head, imported from the COMSOL Multiphysics library, was minimally modified from the original geometry provided by IEEE, IEC and CENELEC. The upper part of the head model was divided into five layers, which, from exterior to interior, respectively represent scalp, skull, cerebrospinal fluid (CSF), grey matter and white matter as shown in Figure 2. In this model, the dura, arachnoid, including the pia, were ignored because they are considerably thinner than other layers. CSF itself is a weak scatterer of light, but due to the presence of the highly scattering strands of arachnoid trabeculae that pass through CSF between the arachnoid membranes and the pia, we employed modified optical parameters for the CSF layer that account for the scattering effect of arachnoid trabeculae[4]. The optical properties of different tissue layers in the presence of IR light at the wavelength of $850 \mathrm{~nm}$ were obtained from previous studies described by [4-6] (Table 1). The light sources, represented by $0.4 \mathrm{~cm}$ diameter illumination spots, are positioned on scalp at the inter-unit distance of 1-3 cm (Figure 3).
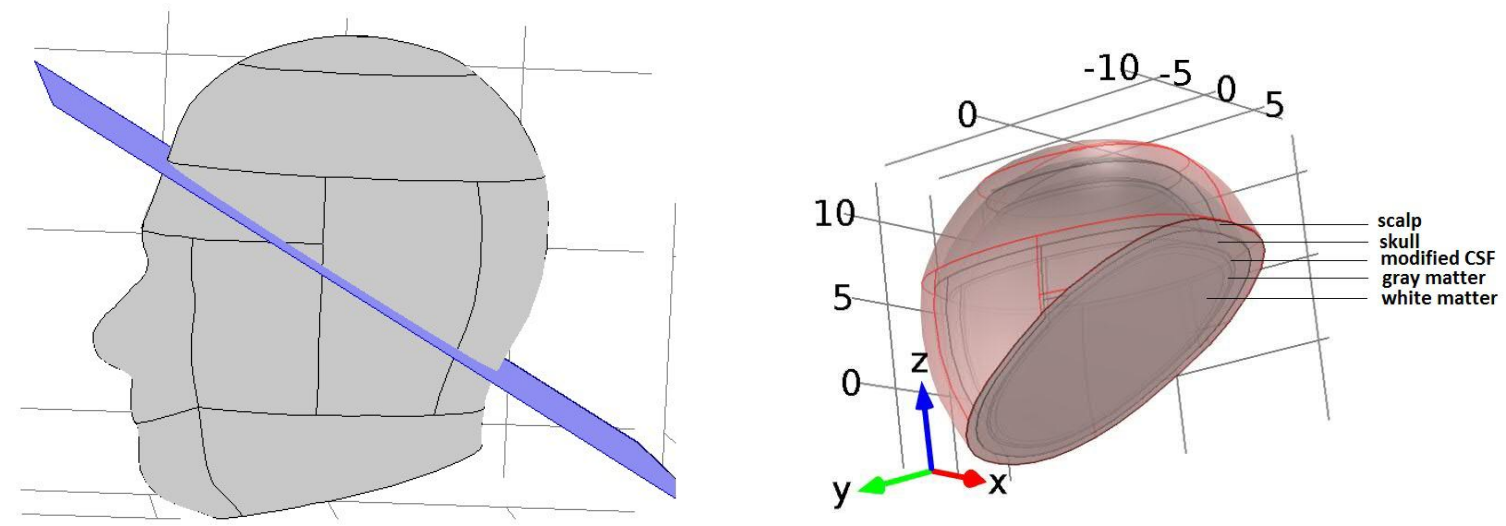

Figure 2. Left: Geometric model of a human head intercepted by a tilted x-y plane. Right: Layered tissues of the upper head model. 


\begin{tabular}{|l|l|l|}
\hline Tissue Type & $\boldsymbol{\mu}_{\mathrm{a}}\left(\mathbf{m m}^{-\mathbf{1}}\right)$ & $\boldsymbol{\mu}_{\mathrm{s}}{ }^{\prime}\left(\mathbf{m m}^{-\mathbf{1}}\right)$ \\
\hline Scalp & 0.012 & 1.8 \\
\hline Skull & 0.025 & 1.6 \\
\hline Modified CSF & 0.009 & 0.8 \\
\hline Gray Matter & 0.036 & 0.9 \\
\hline White Matter & 0.014 & 1.1 \\
\hline
\end{tabular}

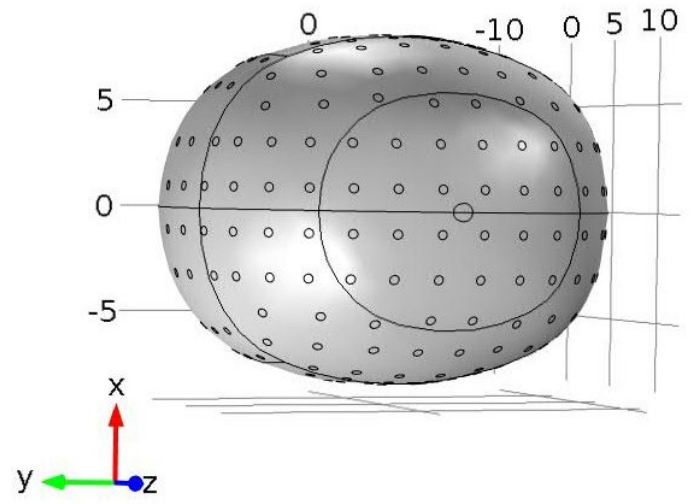

Table 1. Optical properties of the human head model.

Figure 3. A top view of the spatial arrangement of the light sources.

\subsection{Simulation results}

Transcranial penetration of the NIR light emitted by multiple light sources was predicted by the finite element model and the results are illustrated in Figure 4. The left and right panels show slice views of the photon fluence rate profile in horizontal and vertical planes, respectively. In spite of different viewing angles, all slices present similar rainbow-shaped photon fluence color maps that exhibit little variation at similar brain depth, further demonstrating that multi-LED arrays may be used to generate a quasi-radially-symmetric illumination field, regardless of the specific cranial location.

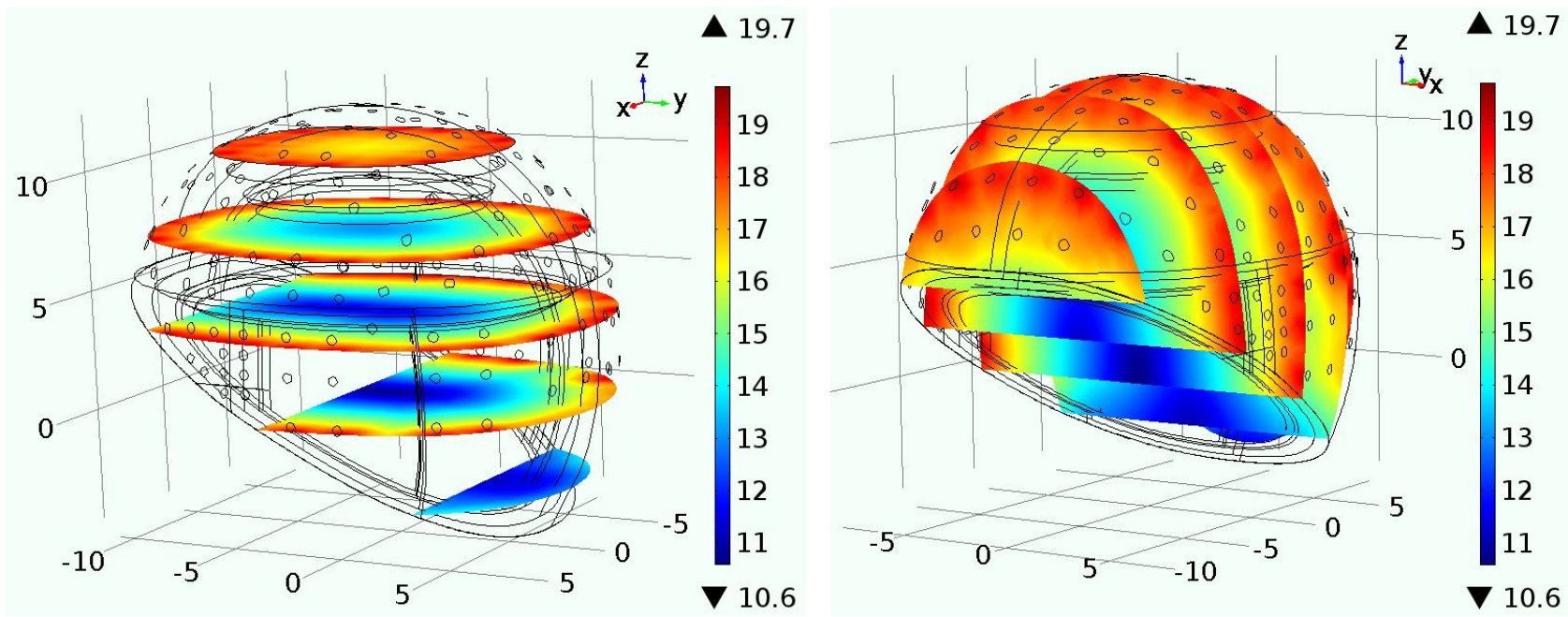

Figure 4. Slice views of photon fluence rate profiles $\left(\log \left(\right.\right.$ fluence rate; $\left.\left.\mathrm{cm}^{-2}\right)\right)$ in horizontal (left) and vertical (right) planes.

In addition, with contributions from multiple sources as simulated above, the transcranial fluence rate undergoes alleviated attenuation. For example, approximately 4-5 orders of magnitude attenuation was exhibited after penetrating 3 $\mathrm{cm}$ deep in the head, reduced by almost an entire order of magnitude from the attenuation of light emitted by a single source alone (Figure 5). Changes in the fluence rate with the penetration depth in the presence of multiple vs. single light source are illustrated in Figure 6. As the light propagated deeper in the brain, multi-source simulation produced progressively decelerated fluence decay, due to strengthened crosstalk between the sources by increasingly extensive photon density superimposition. These results demonstrate the ability of an LED array to generate elevated photon fluence in areas of interest. 

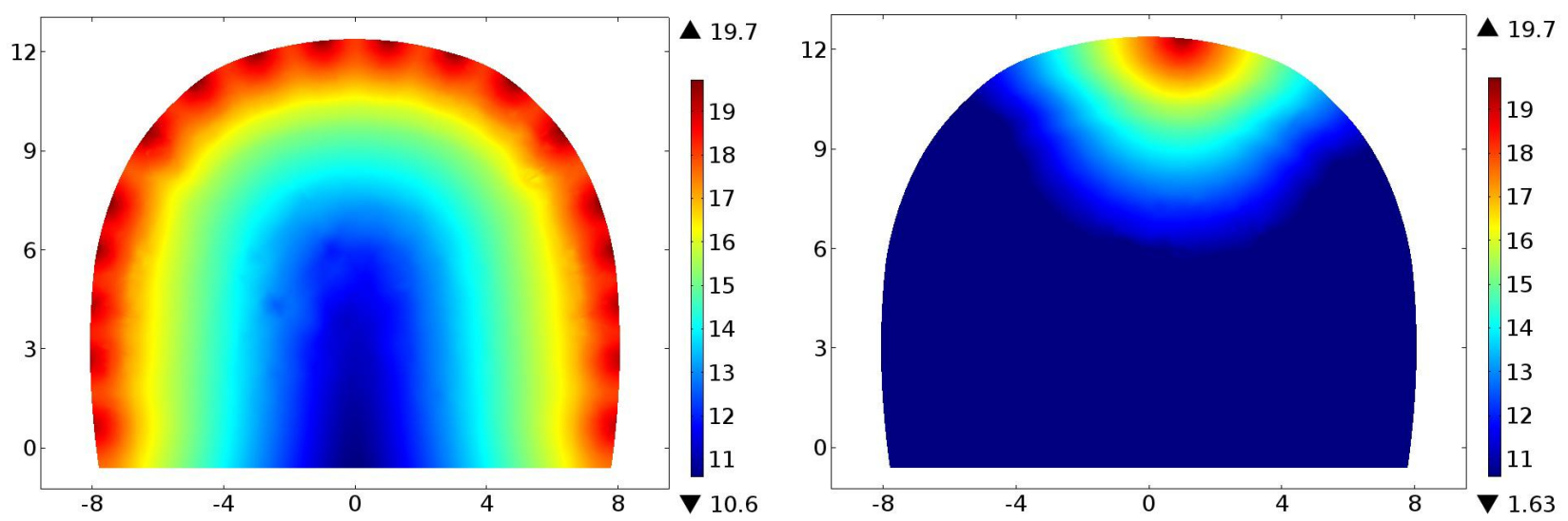

Figure 5. A slice view of $\log$ (fluence rate; $\mathrm{cm}^{-2}$ ) in a frontal-view plane (x-z plane) at $\mathrm{y}=0 \mathrm{~cm}$. Left: simulation obtained with multiple light sources; Right: simulation obtained with only one light source.

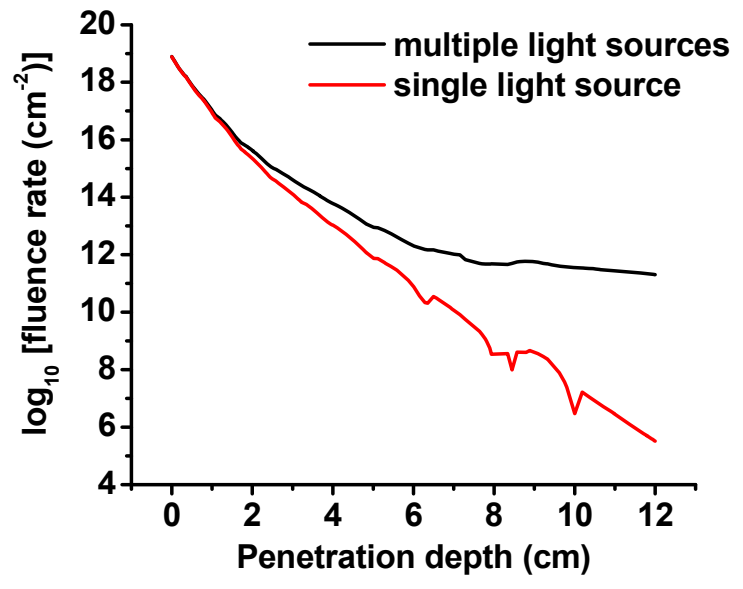

Figure 6. Change of the photon fluence rate with penetration depth along $\mathrm{x}=1 \mathrm{~cm}$ in the Fig.5 plane with multi- vs. single- light source simulation.

\section{THERMAL EFFECT OF THE TRANCRANIAL NIR ILLUMINATION}

To avoid thermal damages to the brain, the illumination system needs to operate in a range such that the maximal permissible temperature rise does not $>1^{\circ} \mathrm{C}$; international limit for bioelectronics implants. To investigate the thermal safety of the use of NIR LED arrays for transcranial illumination, we explored the thermal effect produced by the prolonged illumination with our high power $850 \mathrm{~nm}$ NIR LED emitter on the brain tissues.

We measured the radiation-induced temperature change in the brain excised from the occipital, frontal, temporal and parietal regions (dissection methods and tissue orientation similar to those described in 2.1). Reconstructed tissue blocks were placed in the sample holder, i.e., a glass beaker with a slot on the side, through which a thermometer probe was delivered into the brain. The LED emitter was aligned with the radial center of the beaker, with the emitter-scalp distance of about $4 \mathrm{~cm}$. The thermometer probe was inserted into the brain horizontally, at about $1 \mathrm{~cm}$ below the dura, and the tip was aligned with the emitter vertically.

Our data shows that, during the entire prolonged illumination (300 s), the $850 \mathrm{~nm}$ NIR radiation produced very little thermal effect on the brain tissue in all four tested regions. As illustrated in Figure 7, at the end of the $300 \mathrm{~s}$ illumination, the average increase in the measured brain temperature was less than $0.2^{\circ} \mathrm{C}(\mathrm{n}=3)$, far below the $1^{\circ} \mathrm{C}$ safety limit and not 
significantly different from the non-radiated control samples. Since the tests were not conducted in an enclosed system with finely controlled environmental parameters, the results were prone to the influence of the ambient temperature. As suggested by the results obtained from the control, even in the absence of any NIR illumination, small changes in tissue temperature were observed $(n=4)$, likely reflecting the fluctuation in the room temperature. Overall our data shows that, under the current experimental conditions, the thermal effect produced by NIR radiation from a single high-power LED emitter is negligible, encouraging future measurement with multiple LEDs in an array. In addition, in real applications, LEDs will most likely be configured for shortened illumination periods or time-gated pulsed illumination, further reducing the risks of thermal damages to the brain.

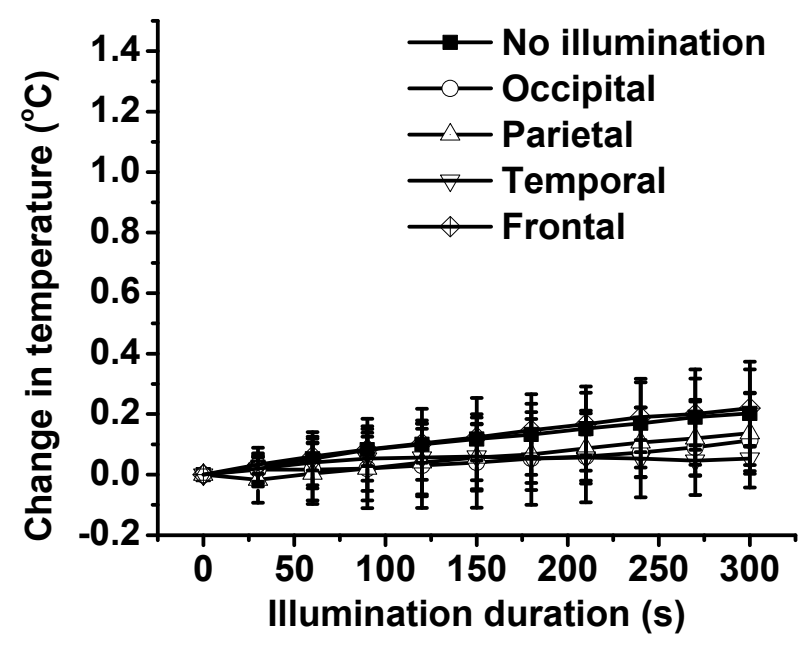

Figure 7. Changes in temperature of the cadaveric brain tissues during prolonged LED illumination (495 $\mathrm{mW}, 300 \mathrm{~s})$.

\section{CONCLUSION}

Highly scattering human head tissues largely limits the effective penetration depth of NIR light. Here we propose the use of a multi-LED array to increase the photon flux in the brain, while keeping the output power from each single LED emitter under the safe limit. With finite element analysis, we demonstrated a quasi-radially symmetrical photon distribution in the brain created by the emitter array. Furthermore, the photon fluence at a given penetration depth is significantly enhanced from that produced by a single emitter alone. In addition, we showed that the temperature of cadaveric brain tissues remain stable under prolonged NIR illumination, suggesting the thermal safety of the LED array.

\section{REFERENCES}

1. Zivin, J.A., et al., Effectiveness and safety of transcranial laser therapy for acute ischemic stroke. Stroke, 2009. 40(4): p. 1359-1364.

2. Naeser, M.A., et al., Improved cognitive function after transcranial, light-emitting diode treatments in chronic, traumatic brain injury: two case reports. Photomedicine and laser surgery, 2011. 29(5): p. 351-358.

3. Schweiger, M., et al., The finite element method for the propagation of light in scattering media: boundary and source conditions. Medical physics, 1995. 22(11): p. 1779-1792.

4. Okada, E. and D.T. Delpy, Near-infrared light propagation in an adult head model. I. Modeling of low-level scattering in the cerebrospinal fluid layer. Appl Opt, 2003. 42(16): p. 2906-14.

5. $\quad$ Okada, E., et al., Theoretical and experimental investigation of near-infrared light propagation in a model of the adult head. Appl Opt, 1997. 36(1): p. 21-31.

6. Jacques, S.L., Optical properties of biological tissues: a review. Phys Med Biol, 2013. 58(11): p. R37-61. 